\title{
Gamma-ray Observations of Blazars with VERITAS and Fermi
}

\author{
Anna Cannon*广 \\ University College Dublin \\ E-mail: annacannon1984@gmail.com
}

\begin{abstract}
VERITAS (the Very Energetic Radiation Imaging Telescope Array System) is an array of four 12m Imaging Atmospheric Cherenkov Technique (IACT) telescopes, located at the Fred Lawrence Whipple Observatory in Southern Arizona. The array has been fully operational since September 2007 and the study of blazars is one of the VERITAS collaboration's key science projects. This paper provides a brief description of both the VERITAS and Fermi Large Area Telescope (Fermi LAT) experiments and an overview of VERITAS' blazar observations to date, highlighting the contemporaneous data from Fermi LAT and other multiwavelength observations where available. Accompanying this is a brief summary of the First Large Area Telescope AGN Catalog and a discussion of recent joint VERITAS-Fermi LAT papers.
\end{abstract}

8th INTEGRAL Workshop "The Restless Gamma-ray Universe"

September 27-30 2010

Dublin Castle, Dublin, Ireland

\footnotetext{
*Speaker.

${ }^{\dagger}$ presented on behalf of the VERITAS and Fermi LAT collaborations.
} 


\section{Introduction}

Active Galactic Nuclei (AGN) are galaxies whose central cores, believed to be supermassive black holes surrounded by bright accretion disks, greatly outshine the rest of the host galaxy. These compact and extremely luminous objects radiate core-dominated, highly variable emission across the electromagnetic spectrum, ranging from the radio to $\gamma$-ray bands. In many cases, highly collimated, relativistic jets are produced, emerging in opposite directions to each other from the accretion disk, and accelerate particles to very high energies. AGN are characterized by this non-thermal emission and a spectral energy distribution (SED) containing two peaks. The peak at low energies is interpreted as synchrotron radiation, while the higher energy peak is generally assumed to be created either by Inverse Compton scattering by relativistic electrons or by hadronic interactions, such as pion decay.

Blazars, believed to be the most powerful and variable subset of AGN, have one jet pointed (within a few degrees) towards the earth. They are classified as either Flat Spectrum Radio Quasers (FSRQs) and BL Lacs depending on the presence of emission lines in their optical spectra. The Fermi LAT collaboration classify a blazar as a BL Lac if it exhibits few or no emission lines, if the equivalent width of the strongest emission line is $<5 \AA$ and the optical spectrum shows a CaII H/K break ratio $<0.4$ [1]. This small CAII $\mathrm{H} / \mathrm{K}$ break ratio ensures that the radiation is predominantly non-thermal. BL Lacs are then further divided into sub-classes depending on the position of the synchrotron peak in its SED. The synchrotron peak for the Low-frequency BL Lacs (LBLs) is located between $10^{13}-10^{15} \mathrm{~Hz}$, for Intermediate-frequency BL Lacs (IBLs) between $10^{15}-10^{16} \mathrm{~Hz}$ and for High-frequency BL Lacs (HBLs) between $10^{16}-10^{19} \mathrm{~Hz}$. This subdivision is believed to be a continuous distribution. In-depth analysis of gamma-ray emission from blazars aids in answering theoretical questions regarding the nature of sources, particle acceleration and the production of cosmic rays.

\section{VERITAS}

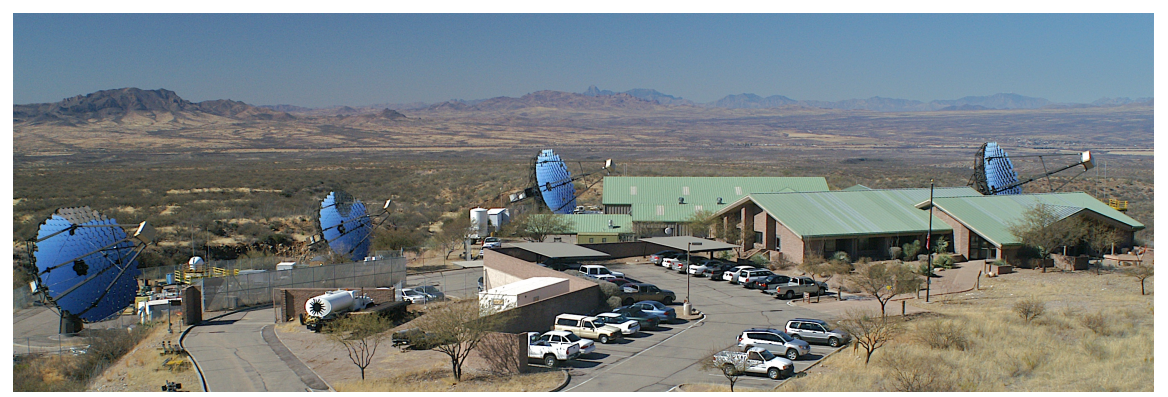

Figure 1: The arrangement of the VERITAS array prior to the relocation of telescope 1 in August 2009.

Currently, the VERITAS collaboration consists of more than 90 members from 22 institutions from Canada, the U.S.A., Ireland, England and Germany. The array (see Figure 2) is the successor to, and its design based on, the pioneering Whipple 10m IACT telescope, which first detected the Crab Pulsar Wind Nebula, the first TeV source, in 1989 [2]. 
VERITAS operates in the energy range $100 \mathrm{GeV}-30 \mathrm{TeV}$ [3]. The energy resolution (above $200 \mathrm{GeV}$ ) is $15 \%-20 \%$ with an angular resolution (68\% containment) of $0.1^{\circ}$. The telescopes are arranged with a baseline of $\sim 100 \mathrm{~m}$ and can detect, at a $5 \sigma$ level, a $1 \%$ Crab Nebula flux source within 26 hours. Cherenkov light from extensive air showers is captured by a 499-pixel photomultiplier camera (located in the focal plane of each telescope) each having a $3.5^{\circ}$ field of view, adequate for both point and diffuse source observations. The array performs stereoscopic observations, using the intersection of the major axes of the image to determine shower core location. In summer 2009, the array underwent an upgrade which consisted of a more efficient mirror alignment technique and the relocation of telescope 1 (to expand the collection area of the array). The results of this upgrade are better image definition and increased flux sensitivity, almost halving the time needed to detect a $1 \%$ Crab Nebula source (see Figure 2). Further upgrades including improvements to the array-level trigger, atmospheric monitoring, camera PMTs and slew speed are due to take place in the near future.

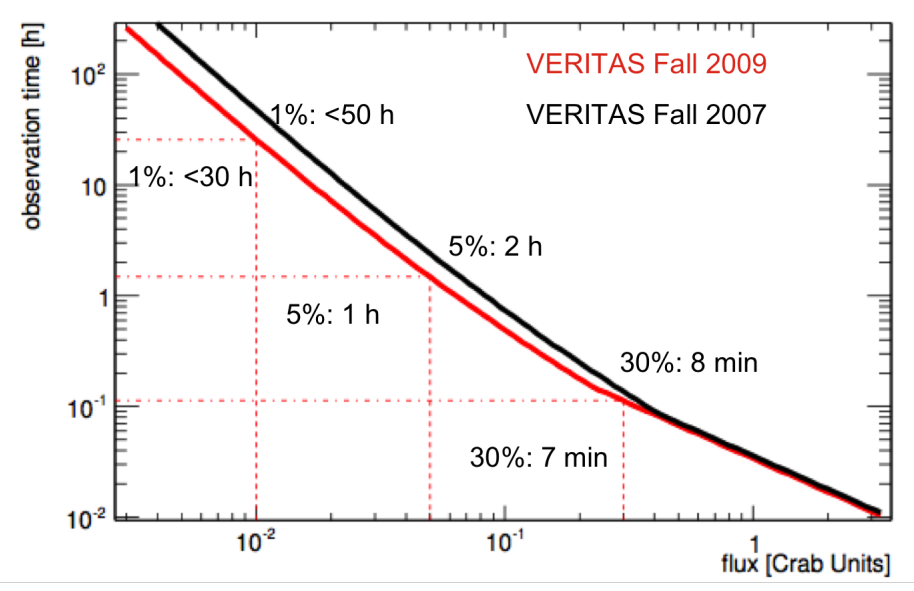

Figure 2: A comparision of VERITAS' sensitivity to the Crab Nebula before and after the relocaton of telescope 1 and the introduction of an improved mirror-alignment technique.

\section{Fermi LAT}

The Large Area Telescope (Fermi LAT) is the primary instrument on-board the Fermi Gammaray Space Telescope, formerly known as GLAST. The successor to EGRET, Fermi was launched on June 11th 2008 and started taking scientific observations on the 4th August 2008. The data have been publicly available for use, along with the analysis software, since August 2009. The LAT collaboration consists of more than 400 members from more than 90 universities and laboratories in 12 countries.

Fermi LAT's two observational modes are survey and pointed with the primary mode being survey. The instrument has a large field of view of $2.5 \mathrm{sr}$ corresponding to $20 \%$ of the entire sky [4]. In survey mode the full sky is observed every three hours in 2 orbits in a zenith-pointing mode, rocking at $35^{\circ}$ north and south of zenith on alternating orbits. The LAT can be used in pointed mode for Targets of Opportunity (ToOs) and is also repointed autonomously for gamma-ray burst 

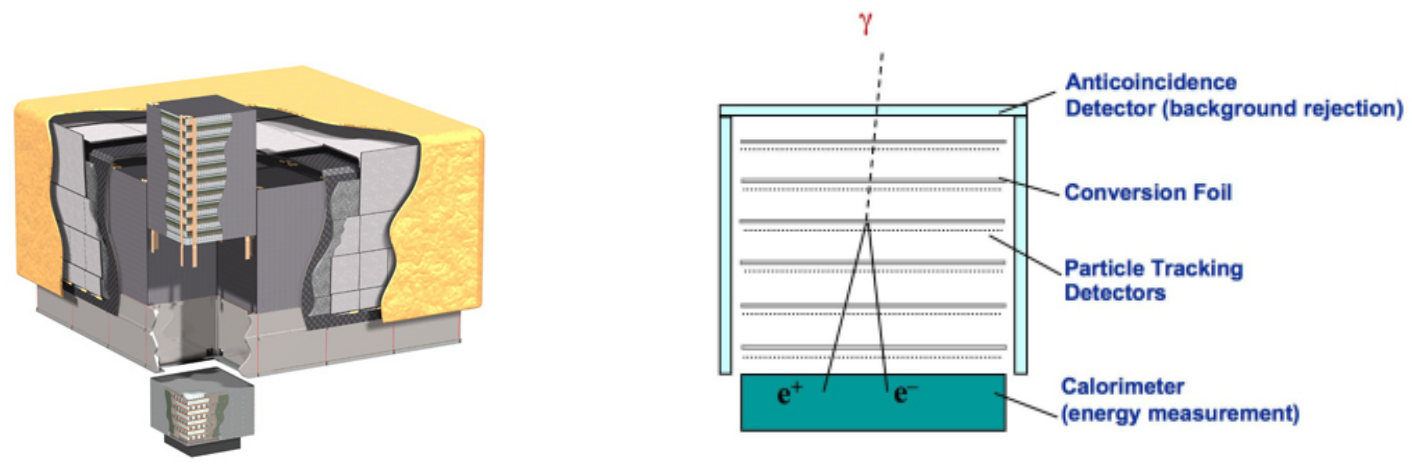

Figure 3: Fermi LAT's tracker, calorimeter and anti-coinicidene detecter.

observations. It is a pair conversion detector operating in the energy range $20 \mathrm{MeV}-300 \mathrm{GeV}$. The high energy range of the LAT intersects with the low energy range of the IACTs so, for the first time, there is an energy overlap between space- and ground-based telescopes allowing continuous spectra between $20 \mathrm{MeV}$ and $30 \mathrm{TeV}$ to be produced. This enables more detailed emission spectral modeling and is thus beneficial in constraining model parameters.

The energy resolution at the highest energies is better than $\sim 10 \%$ and the angular resolution (68\% containment) is $0.1^{\circ}$. The LAT consists of a precision tracker and a calorimeter (to calculate the direction and energy of the gamma ray) and a segmented anti-coincidence system (to reject cosmic rays) along with a programmable trigger and a data acquisition system (see Figure 3). The first year LAT AGN catalog was released in January 2010 and is the result of the first eleven months of observations between August 4th 2008 and July 4th 2009. The catalog contains 1043 1FGL sources at high latitude $\left(|\mathrm{b}|>10^{\circ}\right)$ detected with a $>10 \sigma$ detection. 671 of these gamma-ray sources are associated with 709 AGN, due to multiple associations. Of these 671 sources, 599 are considered "high-confidence" with no multiple associations. 275 of these are BL Lacs, 248 are FSRQs, 26 are "other" AGN (e.g. radio galaxies) and 50 are "unknown" type AGN [5].

\section{VERITAS' recent results}

VERITAS operates an intensive scientific observing program, both galactic and extragalactic, with the in-depth study of blazars as one of the key science projects (ksp). The goals of the blazar ksp include the discovery of new VHE (Very High Energy, $\mathrm{E} \geq 100 \mathrm{GeV}$ ) sources, the long-term monitoring of established VHE sources and the detailed spectral analyses of individual sources thus enabling population and variability studies, the search for flares and more detailed modeling of the gamma-ray emission of blazars. In addition to these goals, the study of distant blazars is important in constraining redshifts and in the study of the extra-galactic background light (EBL). Selection criteria of blazars as targets include sources that are previously known VHE emitters, sources that exhibit a hard Fermi LAT/EGRET spectrum or a hard X-ray spectrum and/or have a sufficiently small redshift. To date, VERITAS has detected 20 AGN, consisting of 13 HBLs, 4 IBLs, 1 radio galaxy (M87, a "misaligned" blazar) and 2 assumed blazars. 10 of these are discoveries, all complemented by multiwavelength data and announced by ATELs. 
In the 2009-2010 observing season the VERITAS blazar catalog was expanded by 6 new sources. All observations (excluding RBS 0413) were motivated by Fermi LAT detections.

\section{Recent joint VERITAS-Fermi LAT papers.}

The subjects of two recent joint VERITAS-Fermi publications, and examples of HBL and IBL spectra are RGB J0710+591 and PKS 1424+240. RGB J0710+591, a HBL with a redshift of $\mathrm{z}=0.125$, was discovered at VHE by VERITAS after being selected as a VHE candidate based on its hard X-ray spectrum. It is the first Fermi-LAT detected source found with the help from VHE instruments and is one of the hardest spectrum VHE blazars known to date with $\Gamma_{\text {VERITAS }}=2.59+/$ $0.26_{\text {stat }}+/-0.20_{\text {sys }}[6]$. The multiwavelength SED is modeled using an equilibrium version of a Synchrotron Self-Compton (SSC) model [7], which includes an Extragalactic Background Light (EBL) absorption model [8], see Figure 4. The interesting result from the SSC model parameters used is that, in order to achieve the observed hard X-ray spectrum, a very hard injection index (1.5) has to be assumed, challenging the standard 1st order Fermi acceleration models. The fit also requires a low magnetic field $(0.036 \mathrm{G})$, far below equipartition. This is not remedied either by increasing the Doppler Factor or by the addition of an External Component (EC) component.

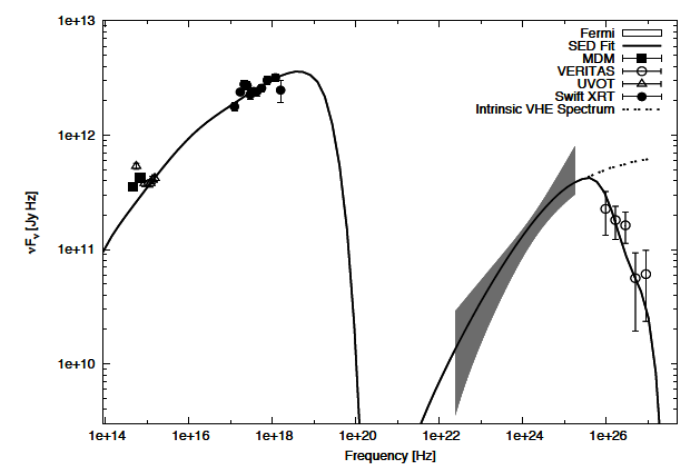

Figure 4: Multiwavelength SED for RGBJ0710+591.

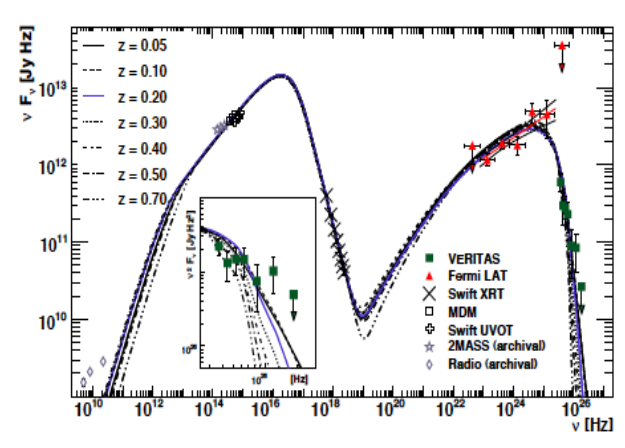

Figure 5: Multiwavelength SED for PKS1424+240.

PKS 1424+241, an IBL with an unknown redshift, was the first VHE source found with the help of Fermi LAT. The VERITAS observations were motivated by the hard Fermi spectrum, $\Gamma_{\text {FermiLAT }}=1.73+/-0.07_{\text {stat }}+/-0.05_{\text {sys }}$ [9]. By extrapolating up from GeV energies, taking into account various EBL models and accompanied by broadband SED modeling, the redshift was constrained to $\mathrm{z}<0.66$ with a $95 \%$ confidence level. Similarly the SED is well fit by an equilibirium version of an SSC model [7], see Figure 5. However above $\mathrm{z} \sim 0.2$, the predicted VHE spectrum becomes increasely sharp compared to the observed VERITAS spectrum. Including an EC component results in a steeper VHE spectrum, leaving the SSC model the better fit.

Both SEDs are fit reasonably well by a purely SSC model where the addition of an EC component does not improve the fit. This indicates that the photons in both peaks are produced by the same source, i.e. with little or no effect from external photons. Depending on the definition used, PKS1424+24, though generally classed as an IBL, can also be classified as a HBL [10]. Therefore, purely SSC emission could be characteristic of this subclass of blazar. In contrast to this, in the 
case of certain IBLs, the inclusion of an EC component (SSC+EC) produces more realistic model parameters, such as Lorentz factor. An example of this is WComae [11] [12].

\section{Conclusion}

Blazar research has advanced rapidly due both to the on-going discoveries from ground-based Cherenkov telescopes and to Fermi LAT producing the most sensitive $\mathrm{MeV}-\mathrm{GeV}$ sky maps to date. With the addition of Fermi LAT data we now have the most detailed measurements of the high-energy peak in the blazar SED. This is essential for constraining model parameters and also for EBL studies. This improved energy coverage, from $\mathrm{MeV}$ to $\mathrm{TeV}$ energies, produces a more accurate picture of the intrinsic properties of blazars and the emission processes taking place. To date, SSC models fit best in the majority of blazars SEDs (in particular HBLs). Further observations and analyses are needed to investigate if this is a property of blazar subclass.

\section{Acknowledgements}

The Fermi LAT Collaboration acknowledges support from a number of agencies and institutes for both development and the operation of the LAT as well as scientific data analysis. These include NASA and DOE in the United States, CEA/Irfu and IN2P3/CNRS in France, ASI and INFN in Italy, MEXT, KEK, and JAXA in Japan, and the K. A. Wallenberg Foundation, the Swedish Research Council and the National Space Board in Sweden. Additional support from INAF in Italy and CNES in France for science analysis during the operations phase is also gratefully acknowledged. This research is supported by grants from the U.S. Department of Energy, the U.S. National Science Foundation, and the Smithsonian Institution, by NSERC in Canada, by STFC in the UK and by Science Foundation Ireland (grants 07/RFP/PHYF271 and 10/RFP/AST2748).

\section{References}

[1] Dermer,C.D., 2010, ArXiv, 1011.0169

[2] Weekes, T. C., et al. 1989, ApJ, 342, 379

[3] Weekes, T. C., et al. 2008, AIP Conf. Prof, 1085, 657

[4] Atwood,W.B., et al. 2009, ApJ, 697, 107

[5] Abdo, A. A., et al. 2010, ApJ, 715, 429

[6] Acciari,V.A., et al. 2010, ApJ, 715, L49-L55

[7] Boettcher,M., \& Chiang,J., 2002, ApJ, 581,127

[8] Franceschini,A., Rodighiero,G., \& Vaccari,M., 2008, A\&A, 487

[9] Acciari,V.A., et al. 2010, ApJ, 708, L100-L106

[10] Padoavani, P., \& Giommi, P., 1996, MNRAS, 279, 526

[11] Acciari,V.A., et al. 2008, ApJ, 684, 73L

[12] Acciari,V.A., et al. 2009, ApJ, 709, 612A 